\title{
Vaginal metastasis of bladder urothelial carcinoma: Description of a case and revision of literature
}

\author{
Carmelo A. Di Franco ${ }^{1}$, Daniele Porru ${ }^{1}$, Giovanni Giliberto ${ }^{1}$, Alessandra Viglio ${ }^{2}$, Bruno Rovereto ${ }^{1}$ \\ ${ }^{1}$ Department of Urology, University Hospital I.R.C.C.S Policlinico S. Matteo, Pavia, Italy; \\ ${ }^{2}$ Department of Pathology, University Hospital I.R.C.C.S Policlinico S. Matteo, Pavia, Italy.
}

\begin{abstract}
Summary Vaginal metastases from urothelial cancer are a rare entity and in literature, few cases are described. We report a case of a 68 year-old woman with history of bladder urothelial carcinoma underwent to radical cystectomy who came in our department after 5 months for pelvic pain and vaginal bleeding. Objective examination revealed an ulcerative, solid vaginal lesion in the upper vaginal wall. We performed a vaginal biopsy that showed urothelial carcinoma compatible with the primitive bladder cancer. The patient underwent to surgery and was sent to oncological evaluation.
\end{abstract}

KEY WORDS: Bladder cancer; Vaginal metastasis; Cistectomy.

Submitted 3 March 2017; Accepted 27 March 2017

\section{INTRODUCTION}

In literature, few cases of vaginal metastases from bladder urothelial carcinoma after radical cystectomy are reported. Usually, urothelial metastases of vagina are a rare entity; in fact, some authors propose preservation of female genitalia organs during radical cystectomy.

We describe a case of vaginal metastasis in a patient with history of bladder urothelial carcinoma who was submitted some months before to radical cystectomy.

\section{Case report}

A 68 years-old woman underwent in June 2016 radical cystectomy with ileal conduit urinary diversion. Pathological report described a bladder neoplastic mass of around 8 centimetres; microscopic histologic examination revealed urothelial carcinoma infiltrating posterior bladder wall (pT2b) high grade (WHO 2004) G2 (WHO 1973), with aspect of squamous differentiation and signs of necrosis, images of lymphatic permeation and negative surgical margins. Vagina specimen was negative for cancer. We performed extended lymphadenectomy (obturator, external and internal iliac, presacral, paraortic and paracaval) with 29 removed lymph nodes all negative for metastases (NO). In November 2016, after five months from cystectomy, the patient accessed in our department for pelvic pain and vaginal bleeding. Objective examination revealed an ulcerative, rough and indolent lesion of anterior vaginal wall on the right side. We performed a cold biopsy of vaginal lesion. Bioptic result suggested an urothelial metastasis of vagina. In the suspect of pelvic disease recurrence, we requested total body CT-scan that showed a vaginal mass of upper right vagina wall. We performed a laborious complete excision of vaginal mass as showed in Figure 1. Definitive pathologic report reported a vaginal white-grey nodular lesion of around $7 \times 6$ centimetres (Figure 2).

\section{Figure 1.}

Surgical specimen of vagina: to note nodular lesion of vaginal wall as it appeared during objective examination.

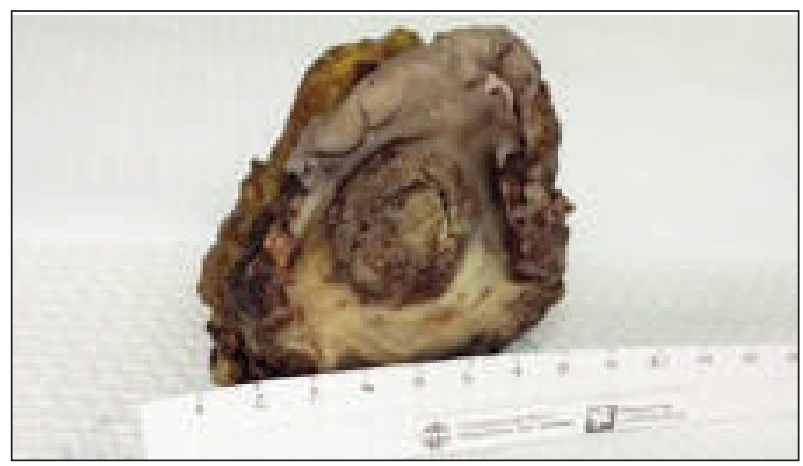

Figure 2.

on cutting the specimen presents white-grey nodular lesion of around 6-7 centimetres.

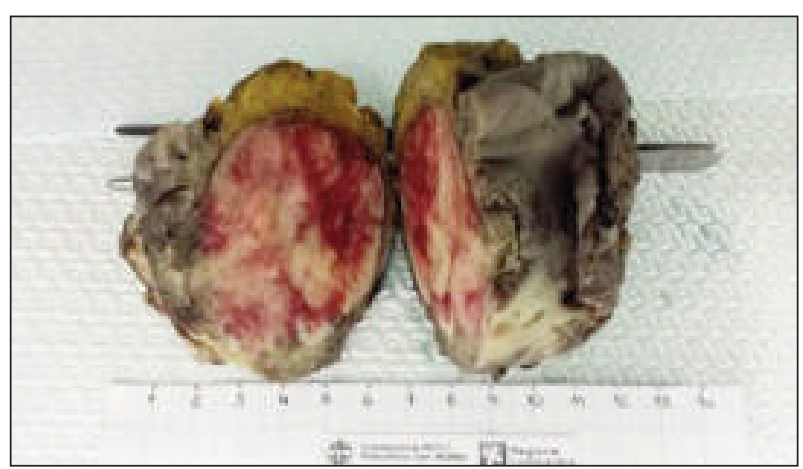

No conflict of interest declared. 
Figure 3.

Microscopically, vaginal lesion was compatible with urothelial carcinoma with extensive signs of squamous metaplasia and necrosis.

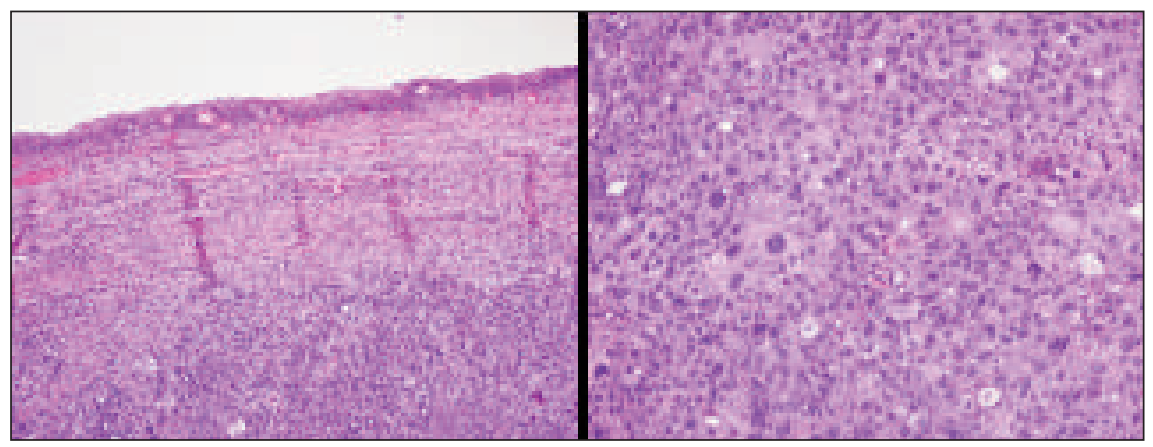

In addition, vaginal specimen was negative for cancer infiltration. We think that metastatic mechanism could be associated to lymphatic permeation through which cancer cells could migrate, not only to lymph nodes but also to adjacent tissues, in this case vagina wall. Probably, also squamous differentiation, because of unknown mechanism, could favour cell implantation in squamous vaginal epithelium. Moreover, the dimension of nodular vaginal lesion (around 6 centimeters) in few months after radical cystectomy,

Microscopically (Figure 3), it was an urothelial metastasis with extensive squamous metaplasia and necrosis. Immunohistochemical examination was cytokeratin 34betaE12+, p63+, cytokeratin 7-, cytokeratin 20-; lesion was compatible with the first bladder cancer reported in radical cystectomy specimen.

\section{Discussion}

Metastasis of urothelial carcinoma to vagina is very rare, with only few cases reported in literature. Literature reports suggest that the risk of urothelial metastases of genital organs after female cystectomy is low (1). In fact, some author propose gynaecologic organs sparing cystectomy in female patient candidate for ileal orthotopic neobladder because it can ensure better urinary continence (2-3). The incidence of secondary malignant involvement of the gynaecological organs by bladder cancer was as low as 2.6-5\% (4). The reported medium time from the diagnosis of bladder urothelial cancer to vaginal metastasis is 4,3 years (5). In 1985, Chin et al. described vaginal recurrence after radical cystectomy; they suggested that the risk of vaginal urothelial metastasis could be previous surgery and radiation to the pelvic organs and patients with tumors other than transitional cell carcinoma, especially those located at the bladder neck and urethra (6). Our patient had no history of pelvic surgery or radiotherapy, bladder cancer was localized in posterior bladder wall, and all removed lymph nodes were negative for metastasis. could suggest that neoplastic cells implanted in vagina wall already before radical surgery. We think that vaginal recurrence after radical cystectomy in a patient with bladder-localized cancer (T2, N0) is a negative prognostic sign that could anticipate a rapid progression of disease.

\section{REFERENCES}

1. Ali-El-Dein B, Abdel-Latif M, Mosbah A, et al. Secondary malignant involvement of gynecological organs at radical cystectomy specimens in women: is it mandatory to remove these organ routinely? J Urol. 2004; 172:885-887.

2. Ali-El-Dein B1, Mosbah A, Osman Y, et al. Preservation of the internal genital organs during radical cystectomy in selected women with bladder cancer: a report on 15 cases with long term follow-up. Eur J Surg Oncol. 2013; 39:358-64.

3. Koie T, Hatakeyama S, Yoneyama T, et al. Uterus-, fallopian tube-, ovary-, and vagina-sparing cystectomy followed by U-shaped ileal neobladder construction for female bladder cancer patients: oncological and functional outcomes. Urology. 2010; 75:1499-503.

4. Chang SS, Cole E, Smith Jr JA, Cookson MS. Pathological findings of gynaecologic organs obtained at female radical cystectomy. J Urol. 2002; 168:147-9.

5. Ohgaki K, Horiuchi K, Oka F, et al. Vaginal metastasis of urothelial carcinoma found incidentally during transurethral resection of a bladder tumor. J Nippon Med Sch. 2008; 75:312-5.

6. Chin Jl, et al. Vaginal recurrence after cystectomy for bladder cancer. J Urol. 1985; 134:58-61.

\author{
Correspondence \\ Carmelo Agostino Di Franco, MD (Corresponding Author) \\ carmelo_difranco@tiscali.it \\ Daniele Porru, MD \\ Giovanni Giliberto, MD \\ Bruno Rovereto, MD \\ Department of Urology Policlinico S. Matteo, Pavia \\ V.le Golgi, 19, 27100 - Pavia, Italy \\ Alessandra Viglio, MD \\ Department of Pathology, University Hospital I.R.C.C.S \\ Policlinico S. Matteo, Pavia, Italy
}

